OPEN ACCESS

Edited by:

Francesca Borrelli,

University of Naples Federico II, Italy

Reviewed by:

James Wolffsohn,

Aston University, United Kingdom

Xuan Zhao,

Shandong University, China

*Correspondence:

Rensong Yue

yuerensong163@hotmail.com

${ }^{+}$These authors have contributed equally to this work and share first

authorship

Specialty section: This article was submitted to

Ethnopharmacology, a section of the journal

Frontiers in Pharmacology

Received: 23 November 2019

Accepted: 15 April 2020

Published: 30 April 2020

Citation:

Yang $M$, Hu Z, Yue R, Yang L, Zhang $B$ and Chen $Y$ (2020) The Efficacy and Safety of Qiming Granule for

Dry Eye Disease: A Systematic Review and Meta-Analysis.

Front. Pharmacol. 11:580. doi: 10.3389/fphar.2020.00580

\section{The Efficacy and Safety of Qiming Granule for Dry Eye Disease: A Systematic Review and Meta-Analysis}

\author{
Maoyi Yang ${ }^{1 \dagger}$, Zhipeng $\mathrm{Hu}^{1+}$, Rensong Yue ${ }^{1 *}$, Liangjun Yang $^{2}$, Boxun Zhang ${ }^{1}$ \\ and Yuan Chen ${ }^{1}$ \\ ${ }^{1}$ Hospital of Chengdu University of Traditional Chinese Medicine, Chengdu, China, ${ }^{2}$ Department of Gastroenterology, \\ Tongde Hospital of Zhejiang Province, Hangzhou, China
}

Background: Dry eye disease (DED) is a common complication in clinical practice. Qiming granule, a traditional Chinese patent medicine, is widely used in treating DED in China. However, its effect is still largely unknown.

Objectives: This research aims to evaluate the efficacy and safety of QG on DED.

Methods: Three English database and four Chinese databases without restriction on language and publication bias were searched. Qualified literature was selecting according to inclusion and exclusion criteria, extracted the data, and conducted a meta-analysis.

Results: A total of 11 articles were included in this meta-analysis. The methodological quality of included studies was low. The results showed that QG was effective for DED (RR:1.26, 95\% Cl:1.12 to $1.41, P=0.0001$ ). The results combined with random effects model showed that QG could significantly prolong the tear film break up time (MD: 2.93, 95\% Cl: 2.22 to 3.65, $P<0.00001$ ), increase the amount of tears in patients with DED (MD: $2.94,95 \% \mathrm{Cl}: 1.83$ to $4.04, P<0.00001$ ) and repair the corneal defects in patients (MD: $-0.71,95 \% \mathrm{Cl}:-1.25$ to $-0.17, P<0.00001)$.

Conclusions: This study found that despite of the apparently positive results of some outcomes, it is premature to confirm the efficacy of QG in treating DED. More high-quality studies are still needed in the future to further confirm the efficacy and safety.

Keywords: qiming granule, traditional Chinese medicine, dry eye disease, systematic review, meta-analysis

\section{INTRODUCTION}

Dry eye disease (DED) refers to a heterogeneous disease mainly involving tears and ocular surface. The occurrence and development of DED are closely related to instability and hyperosmolarity of

\footnotetext{
Abbreviations: DED, Dry eye disease; QG, Qiming Granule; PRISMA, Preferred Reporting Items for Systematic Reviews and Meta-Analyses; CNKI, China National Knowledge Infrastructure; cqvip, VIP information resource integration service platform; RCT, randomized controlled trial; CI, confidence interval; MD, mean difference; RR, risk ratio
} 
tear film (Nelson et al., 2017), ocular-surface inflammation (Sonawane et al., 2012; Tibrewal et al., 2013), sexual hormone imbalance (Schaumberg et al., 2001; Smith et al., 2004; Uncu et al., 2006; Sriprasert et al., 2016), anatomical and neurologic disorders (Tsubota and Nakamori, 1995; Nakamori et al., 1997), compromised neural function (Stern et al., 2004; Mcmonnies, 2017), meibomian-gland dysfunction (Bron and Tiffany, 2004) and other factors like operation and medication (Fraunfelder et al., 2012; Askeroglu et al., 2013; Rosin and Bell, 2013). The clinical symptoms of DED include eye discomfort, light sensitivity, foreign-body sensation, dryness, and irritation, which can cause discomfort and fluctuating vision (Clayton, 2018).

Many therapeutic options are available for DED (American Academy of Ophthalmology (AAO) Cornea/External Disease Panel, 2013). Artificial tear drops treatment is the most widely used treatment and it can provide temporary relief of symptoms. However, according to a review, about $2 / 3$ of patients still have symptoms despite persisting on medication (Downie and Keller, 2015). Side effects including blurred vision, variable ocular discomfort and foreign body sensation can occur (Pucker et al., 2016). Other treatment strategies including reducing inflammation, lifestyle and dietary approaches, treatment of eyelid disease and hormone therapy. However, high quality evidence is still needed to prove the efficacy of these treatments (Jones et al., 2017).

Qiming granule (QG) is a Chinese patent medicine consists of Radix Astragali (huáng qí), Radix Puerariae Lobatae (gé gèn),

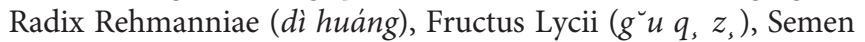
Cassiae (jué ming $z$ ), Fructus Leonuri (chōng wèi $z$,), Pollen Typhae (pú huáng), Hirudo ( $\left.s h u, z h^{\prime}\right)$, which is widely used in the treatment of a variety of eye diseases in China, including diabetic retinopathy, macular edema, DED, and others (Xiangxia et al., 2009; Chao et al., 2019). A series of clinical studies suggested that QG may have a good effect on DED. However, there is no systematic summary on the clinical evidence of QG in the treatment of DED. In this study, in order to confirm the clinical efficacy of QG in the treatment of dry eye disease, the literature on the treatment of DED with QG was systematically collected, and a systematic review and meta-analysis was conducted, hoping to provide evidence-based support for clinical practice.

\section{METHODS AND ANALYSIS}

\section{Study Registration}

This system review and meta-analysis has been registered in PROSPERO website (https://www.crd.york.ac.uk/), an international prospective system review registration website. The registration number is CRD 42018109183. The protocol of this systematic review and meta-analysis has been published (Maoyi et al., 2019). This research was conducted based on this protocol.

\section{Database Search}

The search strategy was in accordance with Preferred Reporting Items for Systematic Reviews and Meta-Analyses (PRISMA) (Moher et al., 2009), and under the consultation with another research (Hu et al., 2019). Six databases including PubMed, Embase, Cochrane Library Central Register of Controlled Trials, China National Knowledge Infrastructure (CNKI) database, Wanfang Data Knowledge Service Platform, and the VIP information resource integration service platform (cqvip) were searched from their inception to November 2019. There were no limitations on language and publication status. In addition, conference articles and clinical registries were also searched for possible related trial. The search was conducted independently by two authors. Detailed search terms can be seen in the protocol previously published (Maoyi et al., 2019).

\section{Inclusion Criteria}

Studies were included if they met all of the following criteria:

1. The studies design was randomized controlled trial (RCT) with parallel control group.

2. The patients in the studies must be diagnosed as DED, whether combined with other diseases or not. There is now a standardized consensus on the diagnosis of DED from TFOS DEWS II focusing on non-invasive testing (Nelson et al., 2017; Wolffsohn et al., 2017). The diagnosis of DED is based on a combination criteria of tear film break-up time test, Schimer test, corneal fluorescein staining and clinical symptoms. There is no restriction on setting of interest and other population characteristics.

3. QG was used as a treatment medicine. If other drugs were used in the treatment group, it must also be used in the control group in the same way.

4. Participants must be adults (older than 18).

5. They must include outcomes that are directly related to DED.

\section{Exclusion Criteria}

Studies were excluded if they met any of the following criteria:

1. The study design was non RCT, such as retrospective study, cross study trial, animal study, case report, and others.

2. For multiple reports from the same study, the one with more details would be retained.

3. Without enough information about the name and use method of the study drug.

4. Papers with insufficient information to conduct a metaanalysis, such as conference papers without data, and others.

\section{Study Selection and Data Extraction}

According to the research protocol, study search, study selection and data extraction was conducted by two reviewers independently and the third reviewer, Rensong Yue, checked the extracted data. A final decision will be made through consensus when there were discrepancies.

\section{Risk of Bias Assessment}

The risk of bias assessment was conducted through the Cochrane collaborative bias risk tool in Review manager 5.3 software by Zhipeng Hu and Maoyi Yang. Any disagree was settled through consultation with the third author Rensong Yue. 


\section{Statistical Analyses}

Statistical analysis was mainly carried out in Review manager 5.3 software and Stata 12.0 software. The $95 \%$ confidence interval (CI) and mean difference (MD) were calculated for the continuous variables and 95\% CI and risk ratio (RR) were calculated for dichotomous variables. $P<0.05$ was considered statistically significant. The heterogeneity of data was investigated by Cochrane $\mathrm{X}^{2}$ and $I^{2}$ tests. The fixed effect model was be used if no significant heterogeneity was observed; otherwise, the random effect model would be applied for statistical analysis. Subgroup analyses were conducted to explore the source of heterogeneity. Publication bias assessment was conducted through funnel plots and Egger's tests if more than 10 trials were included. Sensitivity analysis was used to explore the stability of the results.

\section{RESULTS}

\section{Database Search Results}

A total of 75 studies have been retrieved from six databases, and no grey literature was found in the conference collection and clinical registries. 26 duplicate records were deleted by software. 25 studies were excluded by reading the title and abstract of the literature, including 14 repeatedly published literature, two retrospective studies, two non randomized controlled trials, two case reports, four studies of diseases not DED, one study of patients younger than 18 years old. The full text of the remaining 24 records were downloaded, 13 records were deleted according to the inclusion criteria or the exclusion criteria after full-text reading, and finally 11 studies were included in meta-analysis. A list of records excluded by reading the full text can be found in the Supplementary Material 1. The detailed process of database search is shown in Figure 1.

\section{Study Characteristics}

Eleven RCTs were included in this research, all of which were completed in China (Jinlan and Mingchang, 2013; Jie and Kun, 2014; Xueling et al., 2014; Xingyan and Mingguo, 2015; Chao et al., 2016; Li et al., 2017; Shilin and Hongfang, 2017; Yi and Gaoli, 2017; Mingyue et al., 2018; Qing and Liang, 2018; Siwen, 2019). A total of 1032 DED patients were included in the study. Some studies provided information about number of eyes suffered from DED. However, these studies measured outcomes based on participants, not eyes. Therefore, the information about eyes provided by the researchers does not distort the results actually. Participants ranged in age from 34.3 to 67.6 years old. The average course of disease is 4.7 to 9.0 years. The baseline means of tear break time and Schimer's tear test

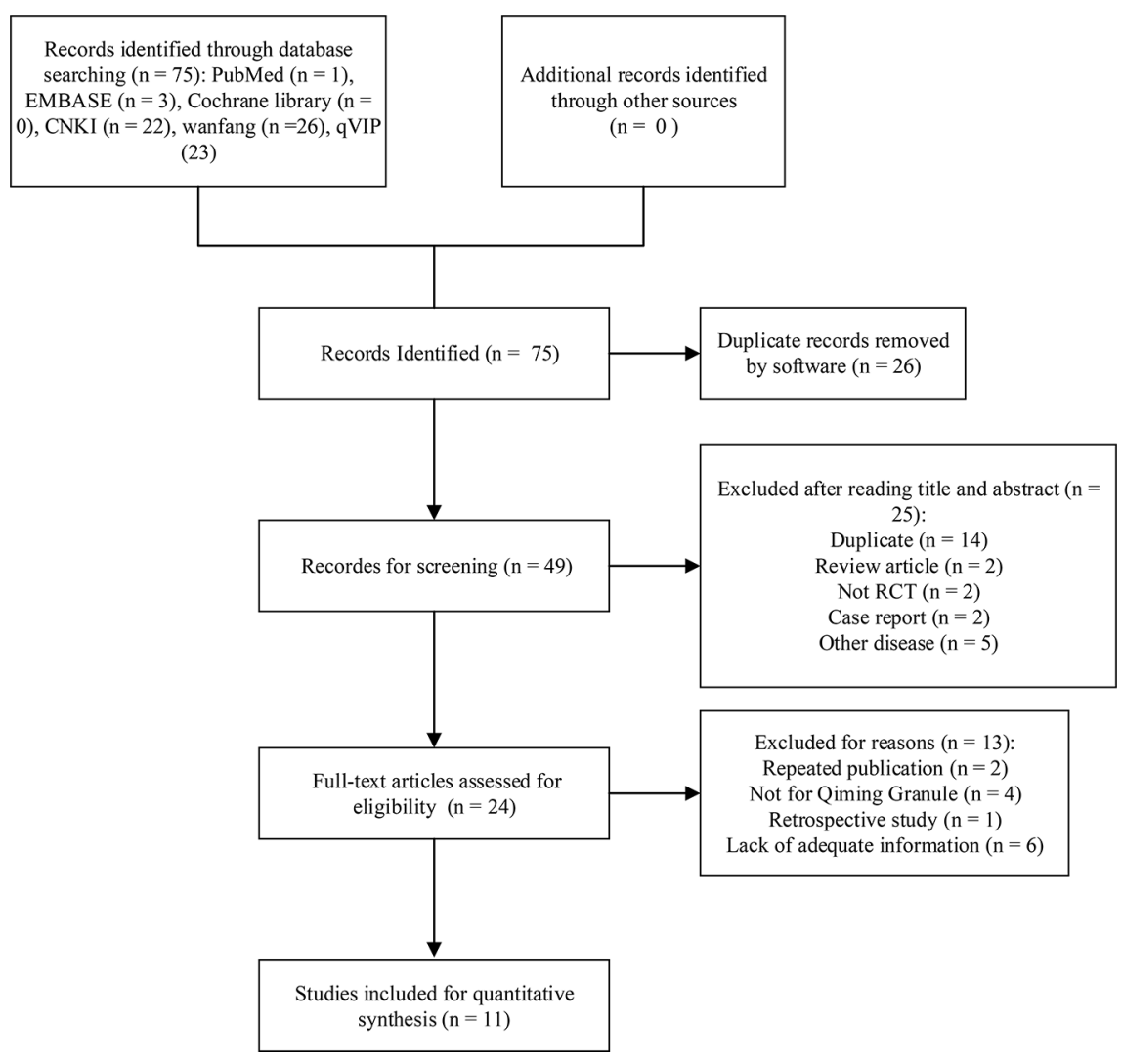

FIGURE 1 | Flowchart of database searching and study identification. 
were 2.51 to $9.02 \mathrm{~s}$ and 2.11 to $6.64 \mathrm{~mm}$, respectively. In most studies, the intervention course was one month, with four RCTs for 2 months and only one RCT for three months. In all the studies included, QG was taken orally by patients at the recommended dose of $4.5 \mathrm{~g} /$ time, three times a day. In three studies, patients in treatment group received QG and patients in control group received conventional treatment. In the remaining eight studies, the patients in control group received conventional treatment while patients in treatment group received a combination treatment of QG and conventional treatment. Therefore, none of these studies were participants masked. The basic characteristics of the studies can be seen in Table 1. All the extracted information is provided in Supplementary Material 2. All QGs used in the included studies were prepared by and purchased from Zhejiang Wansheng Pharmaceutical Co., Ltd. None of the studies gave information about their funding and conflicts of interest. No data on quality control were reported in all the 11 studies.

\section{Risk of Bias Assessment}

In general, the quality of methodology included in the study is not high. Most of the studies did not explicitly report the method of generating random number sequence, and all of them did not clearly state whether the allocation concealment was carried out. In terms of masking method, most of the studies claimed to use blinding method, however, considering that in these experiments, patients in the experimental group received qiming granule orally and patients in the control group were given external eye drops, a high risk in terms of performance bias were determined for these studies. As to detection bias, there were two studies that use objective detection indicators, whose results would not be interfered by the detector, so these two studies were determined as "low risk" while other studies were identified as "high risk". In the aspect of attrition bias and reporting bias, judgement about whether there was reporting bias cannot be made because all the researchers did not register their clinical trial protocol or published protocol before the trial actually started. Similarly, since all the studies had not reported the calculation of sample size in detail, both of them were marked as "unclear risk". All risk of bias assessment data is shown in Figures 2 and 3.

\section{Total Effective Rate}

The total effective rate is a composite end point composed of improvement of clinical symptoms, tear film break up time, Schimer's test and corneal fluorescein staining. The results can be divided into three categories: significantly effective, effective and ineffective. There are some slight differences in the composition of this outcome in different studies. For example, other outcomes may be added to this composite end point in some studies. All the 11 RCTs included in the study compared the total effective rate of QG in DED patients. The results showed that there was significant heterogeneity in total effective rate $\left(P<0.0001, I^{2}=\right.$ $77 \%$ ) (Figure 4). The results showed that QG was effective for DED (RR:1.26, 95\%CI:1.12 to 1.41, $P=0.0001$ ) (Figure 4). Sensitivity analysis indicated that the result was stable (Supplementary Material 3). Subgroup analysis was conducted according to the course of treatment (1 month, 2 months, or 3 months) (Supplementary Material 4) and the intervention (qiming granule alone or combined with other drugs) of the treatment group (Supplementary Material 5). The results of these two subgroup analyses showed that the difference between courses of treatment and concomitant drugs did not lead to heterogeneity and the effective rate of QG would not be different because different courses of treatment and combination of drugs (test for subgroup differences: $P$ all $>0.05$ ) (Table 2).

\section{Tear Film Break Up Time}

All the included articles studied the effect of QG on tear film break up time in patients with DED. The results showed significant heterogeneity $\left(P<0.00001, I^{2}=83 \%\right)$ (Figure 5). The results combined with random effects model showed that QG could significantly prolong the tear film break up time (MD: 2.93, 95\% CI: 2.22 to $3.65, P<0.00001$ ) (Figure 5). Sensitivity analysis indicates that the result is robust (Supplementary Material 6). Subgroup analyses were performed according to different course of treatment (Supplementary Material 7), combination of drugs (Supplementary Material 8), and different baseline levels of tear film break time (Supplementary Material 9). The results of subgroup analysis showed that the effect of QG on prolonging tear film break up time could not be affected by whether patients were treated with QG alone or combined with other drugs and by different baseline levels of tear film break up time (test for subgroup differences: $P$ all $>0.05$ ). However, different course of treatment could affect the effect of QG on the prolongation of tear film break up time (Test for subgroup differences: $P=0.0001$ ) (Table 2).

\section{Schimer's Test}

Nine of the included RCTs reported the results of QG on Schimer's test (Jinlan and Mingchang, 2013; Xueling et al., 2014; Jie and Kun, 2014; Xingyan and Mingguo, 2015; Chao et al., 2016; Li et al., 2017; Shilin and Hongfang, 2017; Mingyue et al., 2018; Siwen, 2019). There was significant heterogeneity in the results of schimer's test $(P$ $<0.0001, I^{2}=95 \%$ ) (Figure 6). The results showed that QG could significantly increase the amount of tears in patients with DED (MD: 2.94, 95\% CI: 1.83 to $4.04, P<0.00001$ ) (Figure 6). Sensitivity analysis indicates that the result is stable (Supplementary Material 10). Subgroup analyses were conducted according to three subgroup hypotheses. The results of subgroup analyses indicated that different courses of treatment (Supplementary Material 11), combination therapies (Supplementary Material 12) and baseline levels (Supplementary Material 13) would affect the results of Schimer's test (test for subgroup differences: $P$ all $<0.05$ ). Comparing the results of different subgroups, it was found that the subgroups with two months of treatment, single use of QG and higher baseline level of patients had better therapeutic effect (Table 2).

\section{Corneal Fluorescein Staining}

Among the RCTs included, three studies reported the effect of QG on corneal fluorescein staining in patients with DED (Jinlan and Mingchang, 2013; Chao et al., 2016; Yi and Gaoli, 2017). The 
TABLE 1 | Characteristics of included studies.

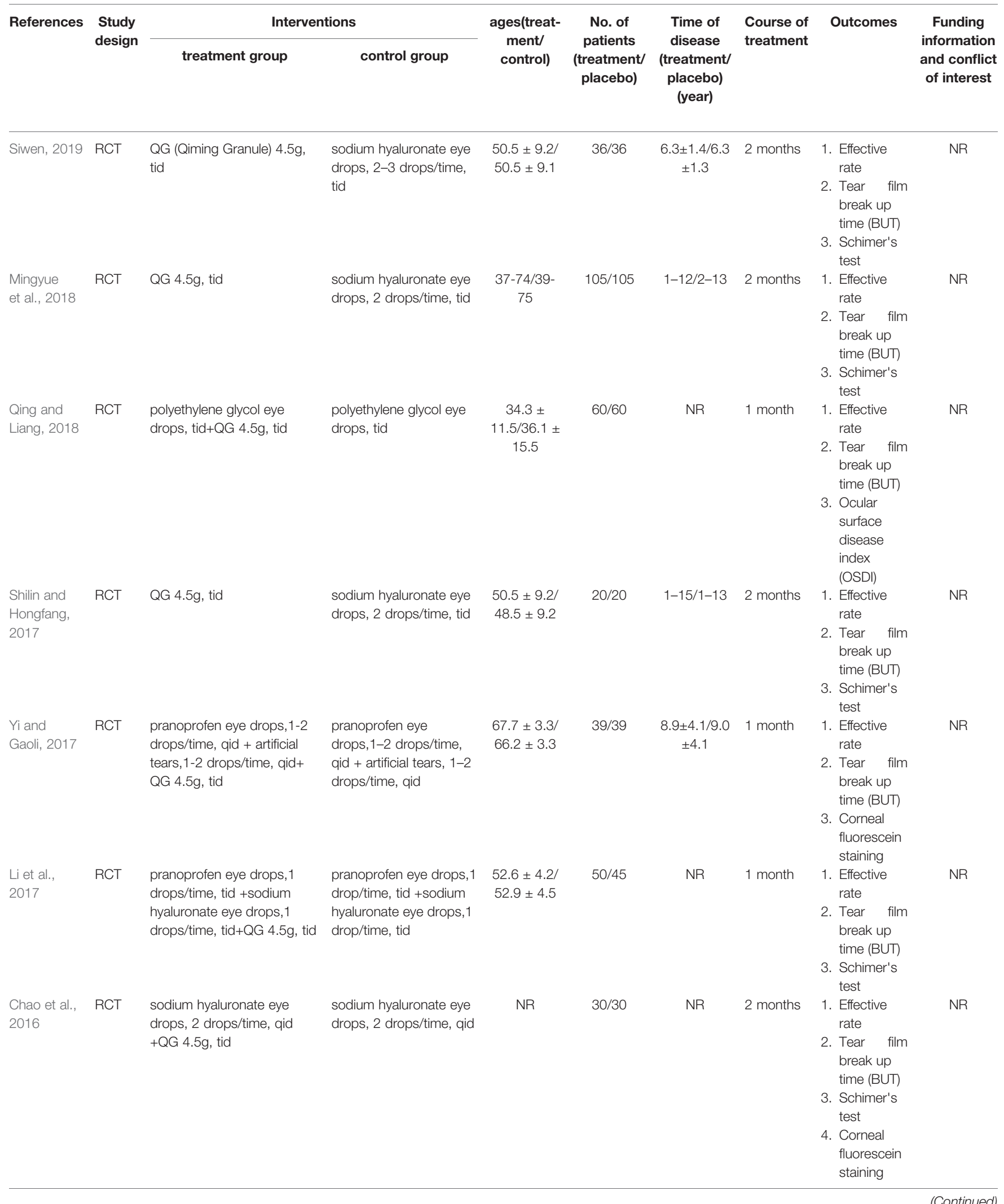


TABLE 1 | Continued

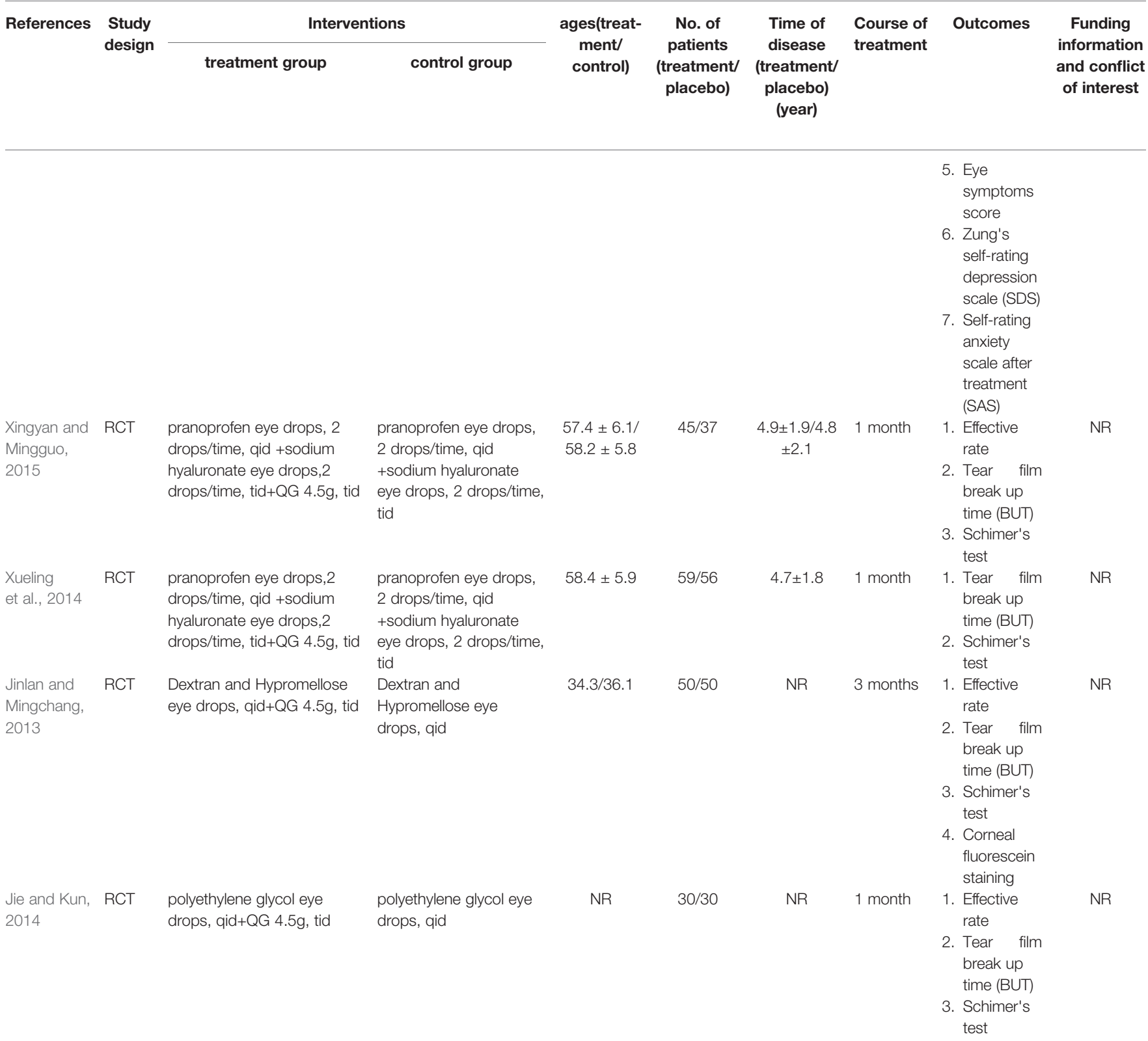

results show that QG can significantly repair the corneal defects in patients (MD: $-0.71,95 \% \mathrm{CI}:-1.25$ to $-0.17, P<0.00001$ ) (Figure 7). However, the results of corneal fluorescein staining showed significant heterogeneity in all studies $\left(P<0.0001, I^{2}=\right.$ 91\%) (Figure 7). Sensitivity analysis indicates that the result is stable (Supplementary Material 14).

\section{Other Results}

Two studies (Chao et al., 2016; Qing and Liang, 2018) reported the effect of QG on the ocular symptoms. One study (Chao et al., 2016) found that ocular symptom scores of DED patients were significantly alleviated $(P<0.05)$ and the other (Qing and Liang, 2018) found a significant reduction in the index of ocular surface. One study reported the effect of QG on the mental state of patients with DED (Chao et al., 2016). The therapeutic effect of QG on the mental state of patients with DED was studied by using Zung's self-rating depression scale (SDS) and self-rating anxiety scale after treatment (SAS). The results showed that QG could improve the tension and anxiety of patients $(P$ all $<0.05)$. One study reported the effects of QG on the levels of MMP-9 (Qing and Liang, 2018). The results showed that QG could significantly improve the level of MMP-9 in tear film and the index of ocular surface disease in patients with dry eye disease.

\section{Publication Bias}

Funnel plot showed the published bias of effective rate (Supplementary Material 15) and tear film break up time (Supplementary Material 16). The funnel plot of effective rate 


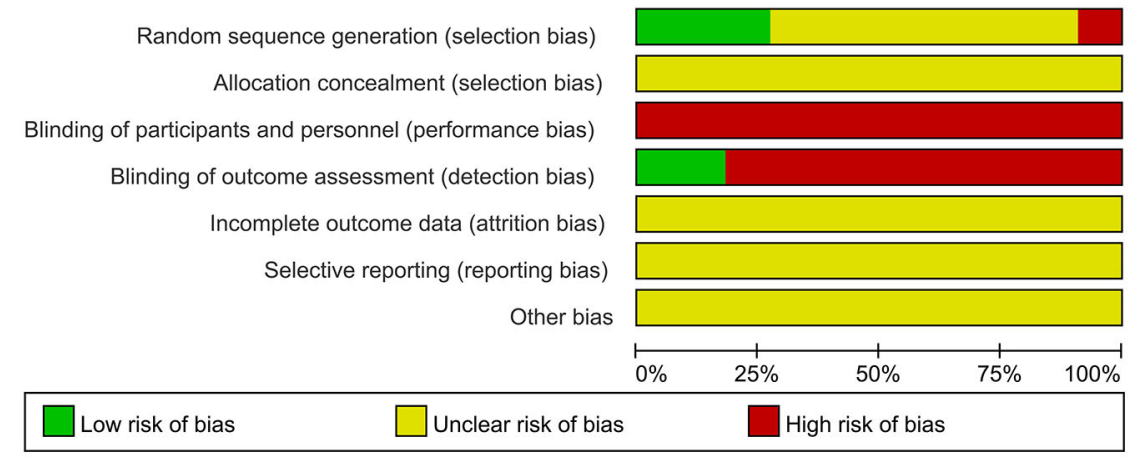

FIGURE 2 | Risk of bias.

was asymmetric, and the funnel plot of tear film break time was symmetric, which means that there was a significant publication bias between the results of effective rate, while there was no significant publication bias between the results of tear film break up time. The results of egger's test were consistent with funnel plot ( $P=0.005$ and 0.117 , respectively). Because the number of RCTs included in Schimer's test was less than 10, there was no funnel chart analysis for this part of data.

\section{Adverse Events}

Of the 11 studies included, two studies mentioned that no obvious adverse events were found during the research (Shilin and Hongfang, 2017; Yi and Gaoli, 2017), while the other nine RCTs did not mention whether any adverse events occurred (Jinlan and Mingchang, 2013; Jie and Kun, 2014; Xueling et al., 2014; Xingyan and Mingguo, 2015; Chao et al., 2016; Li et al., 2017; Mingyue et al., 2018; Qing and Liang, 2018; Siwen, 2019).

\section{DISCUSSION}

In this study, the clinical evidence of QG in the treatment of DED was systemically collated and analyzed so as to provide a better guidance for clinical practice. The analysis of 11 RCTs, indicated that QG can alleviate the clinical symptoms, prolong the break up time of tear film, promote the secretion of tear and repair the damaged cornea, and may have a relieving effect on the depression, and anxiety emotion of patients. However, since there is no effective control in any of the studies, we still cannot rule out whether the efficacy of QG is caused by the placebo effect. Sensitivity analysis showed that the results were stable, but the results were heterogeneous, and there was publication bias in effective rate.

The results of this study have significant heterogeneity and the source of heterogeneity was explored by subgroup analysis. The results showed that different intervention courses would affect the effect of QG on improving tear film break up time. The subgroup analyses of the Schimer's test indicated that the heterogeneity may be caused by the course of intervention, the combination of drugs or not, and the baseline level of patients.
Subgroup analyses of other outcomes failed to find out the source of heterogeneity. Therefore, pre-set subgroup hypotheses did not fully explain the heterogeneity of this study. In terms of effective rate, the scales used in 11 studies were not completely unified, which may be one of the reasons for heterogeneity. There are primary and secondary causes of dry eye disease and the causes of DED in 11 RCTs were different, which may bring heterogeneity to this study. In this study, 11 RCTs were all single center studies, so the regional differences of patients may also be one of the sources of heterogeneity. In addition, there were some defects in the trial design of the included studies, such as no placebo control, no masking method and allocation concealment, and no supervisor to supervise the quality of the trial. All of these factors may lead to the heterogeneity between studies.

DED is mainly caused by damage of corneal surface and decreased tear secretion, and manifested as eye discomfort accompanied by dryness, irritation and fluctuating or blurry vision (Higuchi et al., 2016; Mojzis et al., 2016; Park et al., 2016). Therefore, the time of tear break up and the amount of tear secretion can directly reflect the severity of the disease, so these outcomes are often used in clinical trials. This study found that QG can significantly improve tear break up time and the amount of tear, which suggested that QG can be used in patients with shortened time of tear break up and decreased amount of tear secretion (Kim et al., 2016; Sharma et al., 2016). Previous studies reported that QG was helpful for the treatment of patients with dry eye and corneal epithelial injury which was confirmed in this research. The results showed that QG could repair the damaged cornea of patients with DED. Animal studies had confirmed that QG could increase visual function, protect retinal capillary (Sha, 2013), reduce the concentration of VEGF (Ziming and Bole, 2013) and HIF-1a in retinal tissue, improve the expression level of PEDF protein in retinal tissue, and inhibit the activity of Shh pathway (Lin et al., 2013). This may be the mechanism(s) of QG in repairing corneal injury. However, the mechanism(s) of QG still needs further research.

There are some limitations in this meta-analysis. This study included a small number of RCTs and all of these RCTs were single center, small sample studies. The methodological quality 


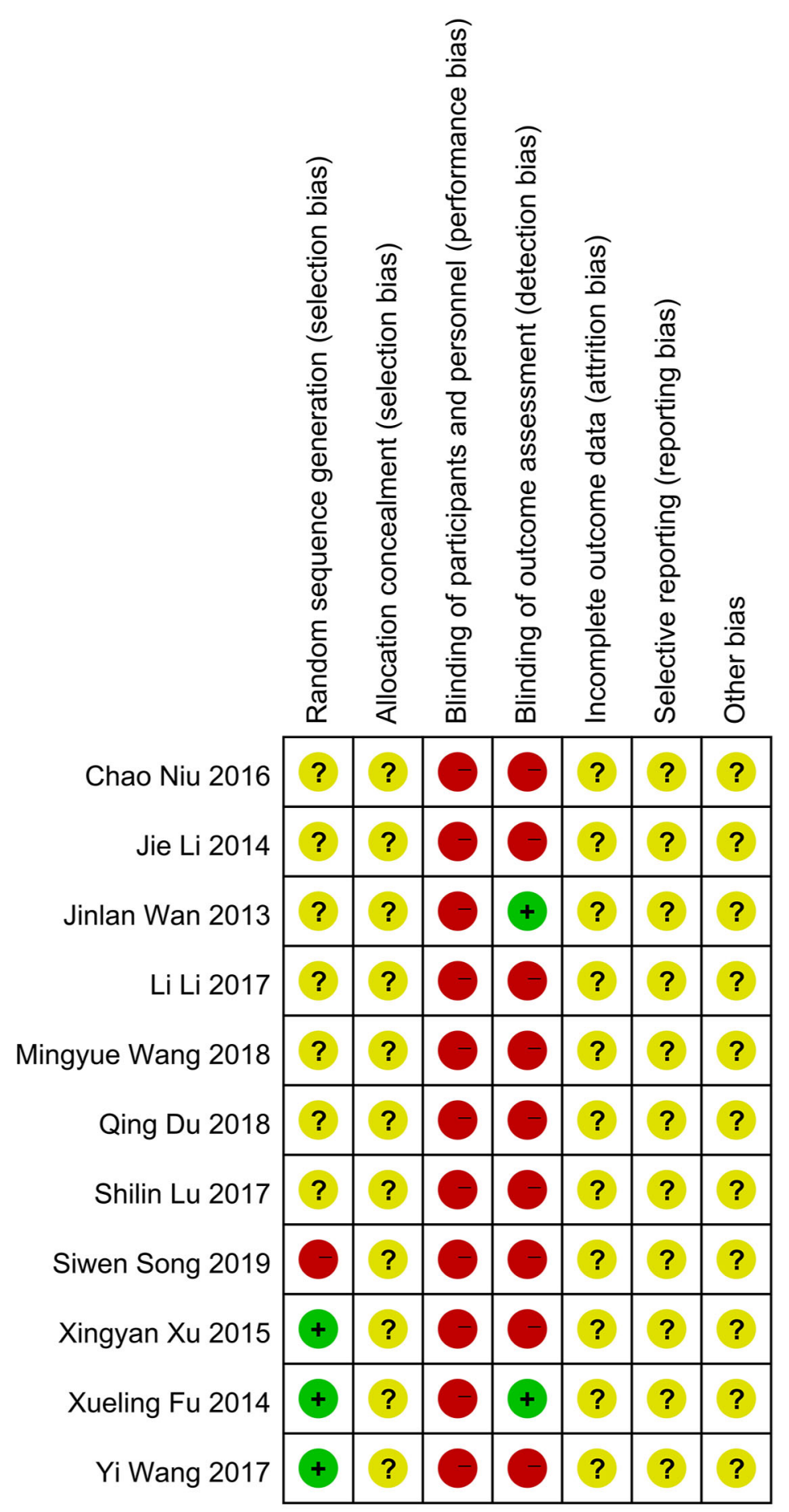

FIGURE 3 | Summary of risk of bias. 


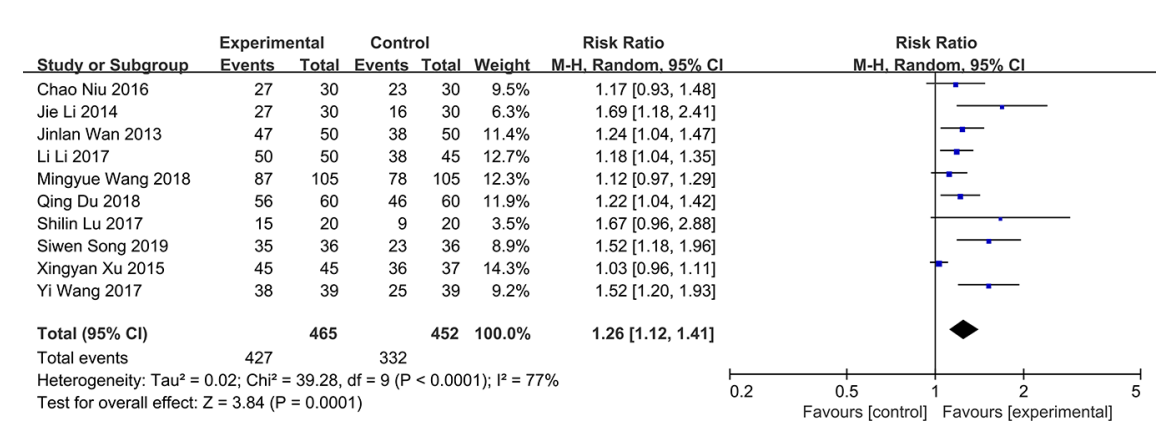

FIGURE 4 | Forest plot for total effective rate.

TABLE 2 | Subgroup analysis for outcomes.

\begin{tabular}{|c|c|c|c|c|c|}
\hline & Number of comparisons & Results & $P$ value for overall effect & $r^{2}$ & $P$ value for subgroup difference \\
\hline Total effective rate & & Risk ratio (95\% Cl) & & & \\
\hline All comparisons & 10 & $1.26(1.12,1.41)$ & 0.0001 & $77 \%$ & \\
\hline Course of treatment & & & & & 0.98 \\
\hline 1 month & 5 & $1.27(1.03,1.56)$ & 0.02 & $89 \%$ & \\
\hline 2 months & 4 & $1.26(1.06,1.50)$ & 0.008 & $49 \%$ & \\
\hline 3 months & 1 & $1.24(1.04,1.47)$ & 0.01 & NA & \\
\hline Intervention of treatment group & & & & & 0.63 \\
\hline Monotherapy & 3 & $1.34(1.02,1.74)$ & 0.03 & $66 \%$ & \\
\hline Combined & 7 & $1.24(1.07,1.43)$ & 0.003 & $82 \%$ & \\
\hline Tear Film Break Up Time & & Mean difference (95\% Cl) & & & \\
\hline All comparisons & 11 & $3.84(2.91,4.76)$ & $<0.00001$ & $65 \%$ & \\
\hline Course of treatment & & & & & 0.0001 \\
\hline 1 month & 6 & $2.59(2.09,3.09)$ & $<0.00001$ & $39 \%$ & \\
\hline 2 months & 4 & $3.84(2.91,4.76)$ & $<0.00001$ & $65 \%$ & \\
\hline 3 months & 1 & $1.12(0.26,1.98)$ & 0.01 & NA & \\
\hline Intervention of treatment group & & & & & 0.23 \\
\hline Monotherapy & 3 & $3.49(2.85,4.13)$ & $<0.00001$ & $0 \%$ & \\
\hline Combined & 8 & $2.81(1.92,3.71)$ & $<0.00001$ & $87 \%$ & \\
\hline Baseline value & & & & & 0.98 \\
\hline High baseline & 4 & $2.90(2.09,3.71)$ & $<0.00001$ & $32 \%$ & \\
\hline Low baseline & 7 & $2.92(1.92,3.91)$ & $<0.00001$ & $89 \%$ & \\
\hline Schimer's Test & & Mean difference (95\% Cl) & & & \\
\hline All comparisons & 9 & $2.94(1.83,4.04)$ & $<0.00001$ & $95 \%$ & \\
\hline Course of treatment & & & & & 0.0002 \\
\hline 1 month & 4 & $1.76(1.11,2.41)$ & $<0.00001$ & $27 \%$ & \\
\hline 2 months & 4 & $4.28(3.27,5.29)$ & $<0.00001$ & $90 \%$ & \\
\hline 3 months & 1 & $2.81(1.24,3.12)$ & $<0.00001$ & NA & \\
\hline Intervention of treatment group & & & & & $<0.0001$ \\
\hline Monotherapy & 3 & $5.00(4.73,5.27)$ & $<0.00001$ & $0 \%$ & \\
\hline Combined & 6 & $1.98(1.55,2.40)$ & $<0.00001$ & $9 \%$ & \\
\hline Baseline value & & & & & $<0.00001$ \\
\hline High baseline & 3 & $5.07(4.73,5.27)$ & $<0.00001$ & $0 \%$ & \\
\hline Low baseline & 6 & $1.97(1.53,2.42)$ & $<0.00001$ & $9 \%$ & \\
\hline
\end{tabular}

NA, not appliable

of these 11 RCTs is not high, which leads to the low level of evidence in this study. None of the studies have an effective control, which may affect the judgement of efficacy. More importantly, since DED is defined by both symptoms and signs, it is critical to analyze the improvement of both symptoms and signs. In the trial included in this study, only one trial analyzed the improvement of patients' symptoms and signs at the same time, so there is still no definite evidence to fully prove the effectiveness of QG in treating DED. In future research, more attention should be paid to development of core outcome set of DED (Wolffsohn et al., 2017).

Both the risk of bias in included studies and risk of bias due to missing results may be influenced by conflicts of interest of study investigators or funders. In this study, all included studies did not clearly report their sources of funding, so judgement about whether there were conflicts of interest cannot be made. These potential conflicts of interest may be the reason of publication bias. In the future research, researchers should report their sources of 


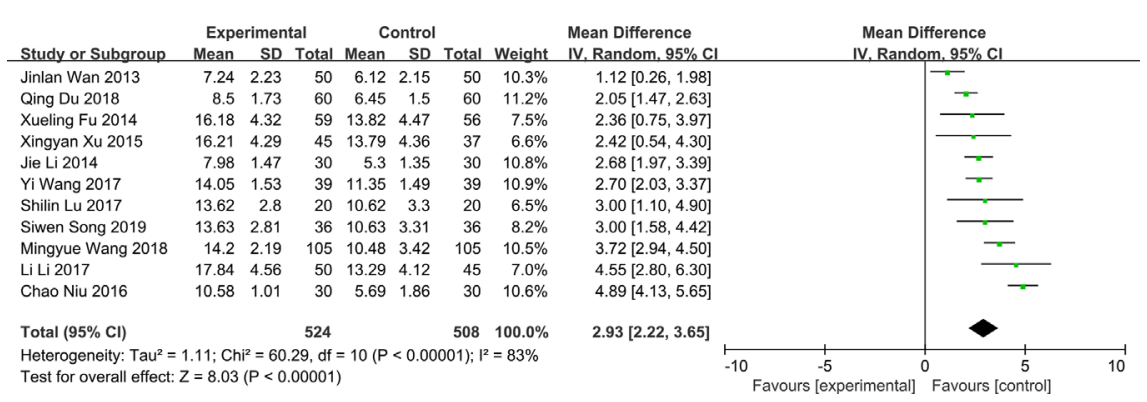

FIGURE 5 | Forest plot for tear film break up time.

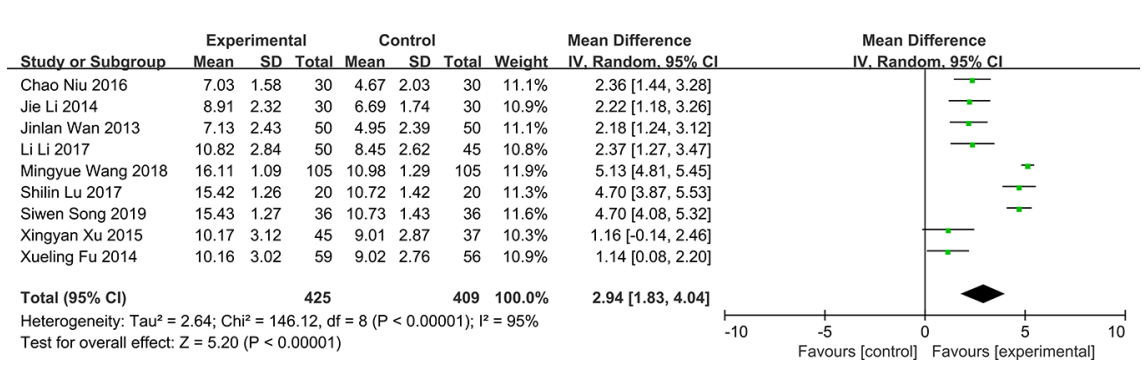

FIGURE 6 | Forest plot for Schimer's test.

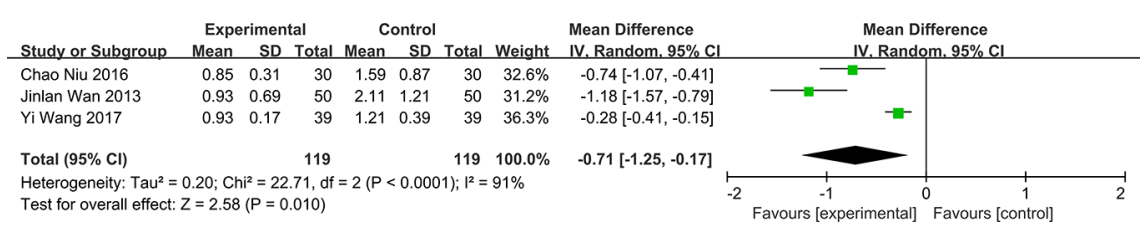

FIGURE 7 | Forest plot for corneal fluorescein staining.

funding and conflicts of interest more clearly, which will be helpful for assessing risk of bias and exploring heterogeneity.

To sum up, QG seems to be effective in improving total effective rate, tear film break up time, schimer's test, corneal fluorescein staining and other outcomes, but there are some defects in the methodological quality currently included and clinician should be cautious when recommending QG in treating DED. More high-quality research is needed to provide high quality evidence for further support of its efficacy.

\section{CONCLUSION}

This study found that despite of the apparently positive results of some outcomes, it is premature to confirm the efficacy of QG in treating DED. More high-quality studies are still needed in the future to further investigate the efficacy and safety.

\section{AUTHOR CONTRIBUTIONS}

The protocol was designed by MY and $\mathrm{ZH}$ under the guidance of RY. This work was conducted by MY, ZH, LY, BZ and YC. The manuscript was drafted by MY and revised by RY and LY. All authors approved the final manuscript before submission. MY and $\mathrm{ZH}$ contributed equally to this work and should be regarded as co-first authors.

\section{FUNDING}

This project was funded by the National Natural Science Foundation of China (No.81774279) and National Science and Technology Planning Project (CN) (2014BAI10B00). The sponsors are not involved in design, execution, or writing the study. 


\section{SUPPLEMENTARY MATERIAL}

The Supplementary Material for this article can be found online at: https://www.frontiersin.org/articles/10.3389/fphar.2020.00580/ full\#supplementary-material

SUPPLEMENTARY MATERIAL 1 | The list of records excluded by reading the full text.

SUPPLEMENTARY MATERIAL 2 | The basic characteristics of the studies.

SUPPLEMENTARY MATERIAL 3 | Sensitivity analysis for total effective rate.

SUPPLEMENTARY MATERIAL 4 | Subgroup analysis according to the course of treatment for total effective rate.

SUPPLEMENTARY MATERIAL 5 | Subgroup analysis according to the intervention of the treatment group for total effective rate.

SUPPLEMENTARY MATERIAL6 | Sensitivity analysis for tear film break up time.

SUPPLEMENTARY MATERIAL 7 | Subgroup analysis according to the course of treatment for tear film break up time.

\section{REFERENCES}

American Academy of Ophthalmology (AAO) Cornea/External Disease Panel. Preferred practice pattern for dry eye syndrome. www.aao.org/preferredpractice-pattern/dry-eye-syndromeppp-2013 2013; Vol. (accessed July 14, 2018).

Askeroglu, U., Alleyne, B., and Guyuron, B. (2013). Pharmaceutical and herbal products that may contribute to dry eyes. Plast. Reconstructive Surg. 131, 159167. doi: $10.1097 /$ PRS.0b013e318272a00e

Bron, A. J., and Tiffany, J. M. (2004). The contribution of meibomian disease to dry eye. Ocular Surf. 2, 149-165. doi: 10.1016/s1542-0124(12)70150-7

Chao, N., Yingli, D., Longjiang, C., and Shuyin, L. (2016). Observation on the clinical effect of qiming granule combined with sodium hyaluronate in the treatment of dry eye. J. Aerospace Med. 27, 941-943. doi: 10.3969/j.issn.20951434.2016.08.006

Chao, Q., Yufeng, Y., Zhenliang, Y., Chenglong, W., and Yan, S. (2019). Metaanalysis of qiming granule in the treatment of diabetic macular edema. J. Liaoning Univ. Tradit. Chin. Med. 21, 128-133. doi: 10.13194/j.issn.1673$842 \times .2019 .02 .035$

Clayton, J. A. (2018). Dry eye. New Engl. J. Med. 378, 2212-2223. doi: 10.1056/ NEJMra1407936

Downie, L. E., and Keller, P. R. (2015). A pragmatic approach to dry eye diagnosis: evidence into practice. Optometry Vision Sci. : Off. Publ. Am. Acad. Optometry 92, 1189-1197. doi: 10.1097/opx.0000000000000721

Fraunfelder, F. T., Sciubba, J. J., and Mathers, W. D. (2012). The role of medications in causing dry eye. J. Ophthalmol. 2012, 285851. doi: 10.1155/2012/285851

Higuchi, A., Oonishi, E., Kawakita, T., and Tsubota, K. (2016). Evaluation of treatment for dry eye with 2-hydroxyestradiol using a dry eye rat model. Mol. Vis. 22, 446453. Available online at: http://kns.cnki.net $/ \mathrm{kns} /$ detail $/$ detail.aspx?FileName= SJPDBE33983088742EA74123657B004EA620\&DbName=SJPDLAST_U.

Hu, Z., Yang, M., Xie, C., Gao, H., Fu, X., Xie, H., et al (2019). Effects of qiming granule for diabetic macular edema: A protocol for systematic review and meta-analysis. Med (Baltimore) 98, doi: 10.1097/MD.0000000000017496

Jie, L., and Kun, L. (2014). Clinical observation of polyethylene glycol eye drops combined with qiming granules in the treatment of dry eye. World's Latest Med. Inf. Abstract (continuous Electronic J.) 24, 132-132. doi: 10.3969/ j.issn.1671-3141.2014.24.096

Jinlan, W., and Mingchang, Z. (2013). Clinical observation of qiming granule combined with artificial tear in the treatment of dry eye. Int. J. Ophthalmol. 13, 1925-1926. doi: 10.3980/j.issn.1672-5123.2013.09.62
SUPPLEMENTARY MATERIAL 8 | Subgroup analysis according to the intervention of the treatment group for tear film break up time.

SUPPLEMENTARY MATERIAL 9 | Subgroup analysis according to different baseline levels of tear film break time.

SUPPLEMENTARY MATERIAL 10 | Sensitivity analysis for schimer's test.

SUPPLEMENTARY MATERIAL 11 | Subgroup analysis according to the course of treatment for schimer's test.

SUPPLEMENTARY MATERIAL 12 | Subgroup analysis according to the intervention of the treatment group for schimer's test.

SUPPLEMENTARY MATERIAL 13 | Subgroup analysis according to different baseline levels of schimer's test.

SUPPLEMENTARY MATERIAL 14 | Sensitivity analysis for corneal fluorescein staining.

SUPPLEMENTARY MATERIAL 15 | Funnel plot for effective rate.

SUPPLEMENTARY MATERIAL 16 | Funnel plot for tear film break up time.

Jones, L., Downie, L. E., Korb, D., Benitez-Del-Castillo, J. M., Dana, R., Deng, S. X., et al. (2017). TFOS DEWS II management and therapy report. Ocular Surf. 15, 575-628. doi: 10.1016/j.jtos.2017.05.006

Kim, Y.-H., Jung, J.-C., Jung, S.-Y., Yu, S., Lee, K. W., and Park, Y. J.. (2016). Comparison of the efficacy of fluorometholone with and without benzalkonium chloride in ocular surface disease. Cornea 35, 234-242. doi: 10.1097/ICO.0000000000000695

Li, L., Shuangshuang, Z., and Jinping, C. (2017). Observation on the effect of qiming granule combined with pramiphene on 50 cases of dry eye after hypermastia. $J$. Community Med. 15, 55-56. doi: CNKI:SUN:SQYX.0.2017-02-024

Lin, L., Xiyuan, Z., and Jihan, L. (2013). Expression of VEGF and PEDF in retinal choroid of diabetic rats. Int. J. Ophthalmol. 13, 1754-1757. doi: 10.3980/ j.issn.1672-5123.2013.09.04

Maoyi, Y., Zhipeng, H., Renson, Y., Liangjun, Y., Zhang, B., and Yuan, C. (2019). The efficacy and safety of qiming granule in dry eye disease: protocol for systematic review and meta-analysis. Med. (Baltimore) 98, e17121. doi: 10.1097/MD.0000000000017121

Mcmonnies, C. W. (2017). The potential role of neuropathic mechanisms in dry eye syndromes. J. Optometry 10, 5-13. doi: 10.1016/j.optom.2016.06.002

Mingyue, W., Zhihua, Z., and Fang, F. (2018). Clinical observation of qiming granule in the treatment of dry eye in patients with type 2 diabetes. Electronic J. Clin. Med. Literature 5, 103. doi: 10.16281/j.cnki.jocml.2018.24.089

Moher, D., Liberati, A., Tetzlaff, J., Altman, D. G.The PRISMA Group (2009). Preferred reporting items for systematic reviews and meta-analyses: the PRISMA statement. PloS Med. 6 (7), e1000097. doi: 10.1016 j.jclinepi.2009.06.005

Mojzis, P., Studeny, P., Werner, L., and Piñero, D.. (2016). Late opacification of a hydro-philic acrylic intraocular lens in Europe. Eur. J. Ophthalmol. 26, 24-26. doi: 10.5301/ejo.5000704

Nakamori, K., Odawara, M., Nakajima, T., Mizutani, T., and Tsubota, K. (1997). Blinking is controlled primarily by ocular surface conditions. Am. J. Ophthalmol. 124, 24-30. doi: 10.1016/s0002-9394(14)71639-3

Nelson, J. D., Craig, J. P., Akpek, E. K., Azar, D. T., Belmonte, C., and Bron, A. J.. (2017). TFOS DEWS II introduction. Ocular Surf. 15, 269-275. doi: 10.1016/ j.jtos.2017.05.005

Park, D. H., Chung, J. K., dR, S., et al. (2016). Clinical effects and safety of 3\% diquafosol ophthalmic solution for patients with dry eye after cataract surgery: a randomized controlled trial. Am. J. Ophthalmol. 163, 122-131. doi: 10.1016/ j.ajo.2015.12.002 
Pucker, A. D., Ng, S. M., and Nichols, J. J. (2016). Over the counter (OTC) artificial tear drops for dry eye syndrome. Cochrane Database Syst. Rev. 2, CD009729. doi: 10.1002/14651858.CD009729.pub2

Qing, D., and Liang, L. (2018). Observation and analysis of the clinical efficacy and safety of qiming granule combined with polyethylene glycol in the treatment of dry eye. J. Liaoning Univ. Tradit. Chin. Med. 20, 193-195. doi: 10.13194/ j.issn.1673-842x.2018.02.058

Rosin, L. M., and Bell, N. P. (2013). Preservative toxicity in glaucoma medication: clinical evaluation of benzalkonium chloride-free $0.5 \%$ timolol eye drops. Clin. Ophthalmol. (Auckland NZ) 7, 2131-2135. doi: 10.2147/opth.S41358

Schaumberg, D. A., Buring, J. E., Sullivan, D. A., and Dana, M. R. (2001). Hormone replacement therapy and dry eye syndrome. JAMA 286, 21142119. doi: $10.1001 /$ jama.286.17.2114

Sha, Z. (2013). Clinical observation on the treatment of non-proliferative diabetic retinopathy with qiming granule compared with compound Xueshuantong capsule. PHD thesis. Available online at: http://kns.cnki.net/kns/detail/detail. aspx?FileName $=1013208484$. nh\&DbName $=$ CMFD2013.

Sharma, N., Thenarasun, S. A., Kaur, M., Pushker, N., Khanna, N., Agarwal, T., et al. (2016). Adjuvant role of amniotic membrane transplantation in acute ocular stevens-johnson syndrome: a randomized control trial. Ophthalmology 123, 484-491. doi: 10.1016/j.ophtha.2015.10.027

Shilin, L., and Hongfang, Z. (2017). Clinical analysis of qiming granules in the treatment of dry eye in patients with type 2 diabetes. China Pract. Med. 12, 2426. doi: 10.14163/j.cnki.11-5547/r.2017.19.009

Siwen, S. (2019). Observe the clinical effect of qiming granule on dry eye in type 2 diabetes. Chin. Med. Guide 17, 207. doi: 10.15912/j.cnki.gocm.2019.11.161

Smith, J. A., Vitale, S., Reed, G. F., Grieshaber, S. A., Goodman, L. A., Vanderhoof, V. H., et al. (2004). Dry eye signs and symptoms in women with premature ovarian failure. Arch. Ophthalmol. (Chicago Ill : 1960) 122, 151-156. doi: 10.1001/archopht.122.2.151

Sonawane, S., Khanolkar, V., Namavari, A., Chaudhary, S., Gandhi, S., Tibrewal, S., et al. (2012). Ocular surface extracellular DNA and nuclease activity imbalance: a new paradigm for inflammation in dry eye disease. Invest. Ophthalmol. Visual Sci. 53, 8253-8263. doi: 10.1167/iovs.12-10430

Sriprasert, I., Warren, D. W., Mircheff, A. K., and Stanczyk, F. Z. (2016). Dry eye in postmenopausal women: a hormonal disorder. Menopause (New York NY) 23, 343-351. doi: 10.1097/gme.0000000000000530

Stern, M. E., Gao, J., Siemasko, K. F., Beuerman, R. W., and Pflugfelder, S. C. (2004). The role of the lacrimal functional unit in the pathophysiology of dry eye. Exp. eye Res. 78, 409-416. doi: 10.1016/j.exer.2003.09.003

Tibrewal, S., Sarkar, J., Jassim, S. H., Gandhi, S., Sonawane, S., Chaudhary, S., et al. (2013). Tear fluid extracellular DNA: diagnostic and therapeutic implications in dry eye disease. Invest. Ophthalmol. Visual Sci. 54, 8051-8061. doi: 10.1167/iovs.13-12844
Tsubota, K., and Nakamori, K. (1995). Effects of ocular surface area and blink rate on tear dynamics. Arch. Ophthalmol. (Chicago Ill : 1960) 113, 155-158. doi: 10.1001/archopht.1995.01100020037025

Uncu, G., Avci, R., Uncu, Y., Kaymaz, C., and Develioğlu, O. (2006). The effects of different hormone replacement therapy regimens on tear function, intraocular pressure and lens opacity. Gynecol. Endocrinol. : Off. J. Int. Soc. Gynecol. Endocrinol. 22, 501-505. doi: 10.1080/09513590600917919

Wolffsohn, J. S., Arita, R., Chalmers, R., Djalilian, A., Dogru, M., Dumbleton, K., et al. (2017). TFOS DEWS II Diagnostic Methodology report. Ocul Surf. 15 (3), 539-574. doi: 10.1016/j.jtos.2017.05.001

Xiangxia, L., Junguo, D., Pinzheng, L., Wu, L., Yu, Y., Qiu, B., et al. (2009). Effect of qiming granule on retinal blood circulation of diabetic retinopathy: a multicenter clinical trial. Chin. J. Integr. Med. 15, 384-388. doi: 10.1007/ s11655-009-0384-5

Xingyan, X., and Mingguo, L. (2015). Analysis of the effect of pramiphene and sodium hyaluronate combined with traditional Chinese medicine in the treatment of the sequelae of hypermastia in the elderly. Int. J. Ophthalmol. 15, 2174-2176. doi: 10.3980/j.issn.1672-5123.2015.12.42

Xueling, F., Ying, J., Xiaoxia, Z., Linying, L., Liming, L., Tao, Y., et al. (2014). Treatmentof 59 cases of dry eye and corneal epithelial injury after diabetic hypermastia with qiming granules and praluofen eye drops. Chin. Pharmaceut. 23, 116-117. Available online at: http://kns.cnki.net/kcms/detail/detail.aspx? FileName $=$ YYGZ201424062\&DbName $=$ CJFQ2014.

Yi, W., and Gaoli, G. (2017). Comparison of the therapeutic effects of different drugs on keratoconjunctival xerosis in diabetic patients after hypermastia. Int. J. Ophthalmol. 17, 1303-1306. doi: 10.3980/j.issn.1672-5123.2017.7.27

Ziming, Y., and Bole, W. (2013). The effect of qiming granule on the curative effect of senile simple diabetic retinopathy and serum VEGF and IGF-1. Chin. Modern Doctor 51, 99-100+112. Available online at: http://kns.cnki. net $/ \mathrm{kcms} /$ detail/detail.aspx?FileName $=$ ZDYS201330041\&DbName $=$ CJFQ2013.

Conflict of Interest: The authors declare that the research was conducted in the absence of any commercial or financial relationships that could be construed as a potential conflict of interest.

Copyright (c) 2020 Yang, Hu, Yue, Yang, Zhang and Chen. This is an open-access article distributed under the terms of the Creative Commons Attribution License (CC BY). The use, distribution or reproduction in other forums is permitted, provided the original author(s) and the copyright owner(s) are credited and that the original publication in this journal is cited, in accordance with accepted academic practice. No use, distribution or reproduction is permitted which does not comply with these terms. 